\title{
Supply-side and demand-side cost sharing in deregulated social health insurance: Which is more effective?
}

\author{
Trottmann, Maria ; Zweifel, Peter ; Beck, Konstantin
}

\begin{abstract}
Microeconomic theory predicts that if patients are fully insured and providers are paid fee-forservice, utilization of medical services exceeds the efficient level ('moral hazard effect'). In Switzerland, both demand-side cost sharing and upply-side cost sharing have been introduced to mitigate this problem. Analyzing a panel dataset of about 150,000 adults, we find both types of cost sharing to be effective in curtailing the use of medical services. However, demand-side cost sharing options are preliminarily chosen by individuals in excellent health, causing 'true' cost savings achieved by supply-side cost sharing to be more important.
\end{abstract}

DOI: https://doi.org/10.1016/j.jhealeco.2011.10.004

Posted at the Zurich Open Repository and Archive, University of Zurich ZORA URL: https://doi.org/10.5167/uzh-78155

Journal Article

Accepted Version

Originally published at:

Trottmann, Maria; Zweifel, Peter; Beck, Konstantin (2012). Supply-side and demand-side cost sharing in deregulated social health insurance: Which is more effective? Journal of Health Economics, 31(1):231-242. DOI: https://doi.org/10.1016/j.jhealeco.2011.10.004 


\title{
Supply-Side and Demand-Side Cost Sharing in Deregulated Social Health Insurance: Which Is More Effective?*
}

\author{
Maria Trottmann† Peter Zweifel; Konstantin Beck ${ }^{\S}$
}

Version: June 2, 2011

\begin{abstract}
Microeconomic theory predicts that if patients are fully insured and providers are paid fee-for-service, utilization of medical services exceeds the efficient level ('moral hazard effect'). In Switzerland, both demand-side cost sharing and supply-side cost sharing have been introduced to mitigate this problem. Analyzing a panel dataset of about 150,000 adults, we find both types of cost sharing to be effective in curtailing the use of medical services. However, demand-side cost sharing options are preliminarily chosen by individuals in excellent health, causing 'true' cost savings achieved by supply-side cost sharing to be more important.
\end{abstract}

Keywords: Health insurance, moral hazard, managed care, copayment, two-stage residual inclusion

JEL-Classification: I11; G22; D82

${ }^{*}$ We thank the CSS Institute for Empirical Health Economics for providing data, financial support, and many insights, Randall Ellis, Wynand Van de Ven, Hansjoerg Lehmann, Johannes Schoder, Stefan Pichler, Ute Kunze, Ute Studer-Merkle, Bernhard Keller, Stefan von Rotz, Urs Kaeser, and participants at the 7th European Conference on Health Economics for helpful comments and discussion.

${ }^{\dagger}$ Corresponding author: University of Zurich, Department of Economics, Hottingerstrasse 10, CH-8032 Zurich, maria.trottmann@econ.uzh.ch, +41 446344596

${ }^{\ddagger}$ University of Zurich, Department of Economics

${ }^{\S}$ CSS Institute for Empirical Health Economics, Lucerne and University of Zurich, Department of Economics 


\section{Introduction}

One of the main goals of health care financing systems is to promote efficient levels and types of care (Ellis and McGuire [1993]). A stumbling block is the fact that insured patients might demand larger than optimal quantities, connoted moral hazard. Zeckhauser [1970] and Zweifel and Manning [2000] have analyzed how demand-side cost sharing can be used as a corrective. However, demand-side cost sharing exposes consumers to risk, contradicting the very objective of insurance. Unless limited by a stop-loss feature, it might also make beneficial procedures unaffordable to patients (Nyman [1999]).

These considerations have created interest in the alternative of supply-side cost sharing. Because of their information advantage, providers of medical care can influence the demand for their services to a greater extent than other professionals (Arrow [1963]). If paid feefor-service, they share patients' incentive for too much or too expensive care. Supply-side cost sharing is designed to make them oppose their patients' moral hazard (McGuire [2000]). However, it might also lead to the denial of beneficial but costly services, a phenomenon commonly termed stinting (Newhouse [2002]).

Both demand-side and supply-side cost sharing have been empirically examined in terms of their effectiveness (see section 2). The novelty of this paper is that it directly compares the expenditure effects of demand-side and supply-side cost sharing, using contract variants offered by the same health insurer. This has the advantage that many side conditions (underwriting policy, billing procedure) are kept constant. Moreover, it complements Lehmann and Zweifel [2004], who construct a proxy for unobserved health status from prior HCE, by the two-stage residual inclusion estimator (2SRI, Terza et al. [2008]). In this way, self-selection effects are more fully controlled for. Finally, it extends the set of instruments influencing choice of plan but not HCE by including the premium for the baseline contract, the potential premium reduction for a restricted plan, the individual's credit record, and years of membership with the same fund. For the capitated plan, an additional instrument is a dummy indicating whether or not an independent practice association (IPA) was operative in the county of the individual.

Focusing on expenditure effects, this paper neglects other aspects of patient well-being. However, it is important to note that consumers in the data have annual free choice of contract 
(without pre-selection by employers or government agencies). ${ }^{1}$ If, for example, a managedcare type plan is too restrictive compared to the premium rebate that is offered, it will not be chosen. Similarly, low income individuals are less likely to chose a high deductible, because transfers from health insurance to the ill are most beneficial if they are large compared to income (Nyman [1999]).

The remainder of this article is structured as follows. Section 2 contains an overview of the empirical literature. The policy setting is described in Section 3 while section 4 is devoted to a description of the data base. In Section 5, we explain the econometric methods used to separate moral hazard from self-selection effects and to deal with the very skewed distribution of HCE data. The estimation results are presented in section 6. Section 7 discusses policy implications in view of related literature, while the final section 8 contains a summary and conclusions.

\section{Literature Review}

In order to keep this review concise, we focus on empirical papers that measure moral hazard in health insurance. When individuals have a choice of plan, self-selection effects need to be accounted for because those who expect high future HCE are more likely to opt for more comprehensive insurance. A small number of researchers have avoided this selection problem by benefiting from randomized experiments (the famous RAND study; Manning et al. [1987]) or natural experiments (Chiappori et al. [1998], Eichner [1998], or Winkelmann [2004]). Other papers have used econometric techniques to address endogenous plan choice. Many econometric approaches require for identification the availability of at least one variable that influences contract choice but not utilization (an 'identifying instrument'). Pertinent studies from Switzerland are Schellhorn [2001], Gerfin and Schellhorn [2006] and Gardiol et al. [2006]. The former two rely on premium level and supplementary hospital insurance as identifying instruments, while the latter uses death as an indicator of morbidity which is unaffected by insurance. Using Australian data, Cameron et al. [1988] advocate income as determinant of insurance coverage but not utilization. In the US, employers play a strong role in determining the individual's choice of plan, so they can be used as identifying instruments. For example,

\footnotetext{
${ }^{1}$ Low income individuals, currently about 30 percent of the population, are eligible to premium subsidies.
} 
Dowd et al. [1991] and Cardon and Hendel [2001] exploit the fact that different employers offer different premiums and co-payment levels, while Deb and Trivedi [2009] use the employer's type (public or private), the size of the firm and whether or not the employer offers both HMO and non-HMO options.

Turning to estimation techniques, one notices that instrumental variable estimators are not often applied to non-linear frameworks. An early exception are Dowd et al. [1991] who estimate a tobit model with a correction for selectivity (Lee [1978]). However, their approach requires strict distributional assumptions such as homoscedasticity. Deb and Trivedi [2009] and Deb et al. [2006] also specify a fully parametric model of both choice and utilization equations, which is jointly estimated by maximum simulated likelihood. As health care expenditure data are extremely skewed and the distribution of the 'tail' is difficult to specify correctly, Terza et al. [2008] advocate the two-stage residual inclusion estimator. It yields consistent estimates in a wide range of non-linear least squares settings.

Studies that have addressed endogeneity in non-linear panel data models are even more rare. Non-linear fixed-effects models are plagued by the incidental parameters problem (see Lancaster [2000] for an overview, or Chamberlain [1980] for a corrective). In random effects specifications, the incidental parameters problem can be avoided by integrating out the individual specific effects (Vella and Verbeek [1998] and Vella and Verbeek [1999]). However, this again requires a parametric specification of their distribution.

An alternate approach of exploiting the information of panel data was pioneered by Wolfe and Godderies [1991]. It uses HCE from prior years to proxy unobserved differences between individuals which become predetermined in the year the comparison between plans is performed (Lehmann and Zweifel [2004], Van Kleef et al. [2008]). In this paper, a mixture between the IV and the 'health proxy' approach will be applied.

\section{Swiss Health Insurance}

Swiss health insurance is of the 'managed competition' type (Enthoven [1978], Van de Ven et al. [2007]). Coverage is mandatory for a rather comprehensive 'basic' basket of medical services and pharmaceuticals, written by some 80 private, not-for-profit insurers competing in a regulated market. Free consumer choice of plan is a distinctive feature of the system. 
There is no pre-selection of plans by employers or government agencies and insurers must accept all applicants during annual open enrollment periods. Premium subsidies for lowincome individuals are funded out of general tax. Premiums can be differentiated by area of residence but not by health risk. Reductions are possible for young adults (19-25) and individuals who receive accident coverage through the employer.

In the baseline contract, insured individuals enjoy unlimited access to all licensed physicians and most hospitals in their region of residence. They face a minimum annual deductible of CHF 300 (some EUR 200 as of 2006) and a co-insurance rate of 10 percent up to a cap of CHF 700 (EUR 470) per year. Physicians in independent practice are reimbursed feefor-service according to an administered fee schedule that is collectively bargained between the providers' and the insurers' associations. Hospitals receive per diems for patients treated (the introduction of a DRG system is under way). The cantons ${ }^{2}$ finance hospital investment and one-half of operational cost. While this system is generally found to ensure access to comprehensive health care to all citizens, it is criticized for high and rapidly increasing $\mathrm{HCE}$, lack of co-ordination between providers, and lack of information about quality and efficiency (OECD [2006]).

In response to these problems, insurers have been granted the right to offer managed-care type options (since 1994) and higher deductibles (since 1996) in return for lower premiums. However, policy makers feared that these options would attract low risks. In addition to a risk adjustment scheme based on age and sex, they imposed limits on possible premium reductions. For voluntary deductibles, these are fixed percentages of the base premium or 80 percent of the additional risk (deductible minus 300), whichever is less. The eligible deductible levels are also regulated, as shown in Table 1. In managed-care type contracts, the insurer must prove that the reduction is justified by efficiency gains rather than self-selection effects. Furthermore, it must not exceed 20 percent during the first five years since the launch of the contract. The same deductible levels apply to managed care-type and FFS plans.

\footnotetext{
${ }^{2}$ Switzerland is divided into 26 cantons, with population ranging from 1,307,600 (Zurich) to 15,500 (Appenzell i.R.), Source: Swiss Federal Statistical Office, www.bfs.admin.ch.
} 
Table 1: Regulation of deductibles and maximum premium reductions, 2006

\begin{tabular}{lrrrrrr}
\hline Deductible level in CHF / Year & 300 & 500 & 1,000 & 1,500 & 2,000 & 2,500 \\
Max reduction in percent of the base premium & - & 5 & 15 & 30 & 38 & 43 \\
Max absolute reduction: $0.8 *$ (Deductible - 300) & - & 160 & 560 & 960 & 1,360 & 1,760 \\
\hline CHF 1 $\approx$ EUR 0.66
\end{tabular}

\section{Data}

The data base consists of individual records of more than 160,000 Swiss adults insured by CSS, a major Swiss insurer, and covering the years 2003 - 2006. It includes age, gender, residential location, contract choice, and HCE. Individuals who were not observed over the entire four years are excluded from the analysis, with death constituting the main cause. While the deathbound are known to cause a considerable amount of HCE, they exhibit an idiosyncratic pattern of health care utilization (see Werblow et al. [2007]), justifying separate analysis. The influence of closeness to death also calls for exclusion of individuals who died during 2007.

Table 2 shows descriptive statistics according to contract choice as of 2006. For simplicity, deductibles are grouped into three categories (low: 300, medium: 500, high: $\geq 1,000 \mathrm{CHF}$ per annum). More than 70 percent of those who chose a deductible in excess of CHF 500 opted for the CHF 1,500 level. As the other deductible levels where chosen by a small number of individuals each, including them separately would have lead to considerable instability, especially in the contract choice equation (see section 5). Furthermore, observed HCE across the high deductible levels appeared to be similar. ${ }^{3}$

Buyers of the high-deductible plans are younger and more likely to be male than those with a low deductible. Their mean HCE amounts to CHF 1,057 or 23 percent of that pertaining to individuals with the minimum deductible. The fraction of those reporting positive HCE is 57 rather than 88 percent. If only those with positive HCE are taken into account, the mean is CHF 1,804 or 34 percent of the low-deductible benchmark, respectively. These differences

\footnotetext{
${ }^{3}$ For patients with high deductibles, it is questionable how well their HCE are observed. In earlier work (e.g. Lehmann and Zweifel [2004]), only HCE in excess of the deductible was analyzed on the grounds that patients have no incentive to submit their claims unless HCE exceeds the deductible. However, with the raise of electronic billing systems, the lion's share of billings are now transmitted directly from providers to insurers. The deductible is then billed to the patient by the insurer. In some cantons, physicians even decided to abandon direct-to-consumer billings completely. CSS has conducted an internal study relating the share of direct-to-consumer billing to HCE below the deductible. The variable did not increase the explanatory power of the model. In our dataset, $24 \%$ of those with positive cost had cost below their deductible.
} 
point to sizeable effects of demand-side cost sharing (which still need to be corrected for self-selection effects, see below).

Table 2: Descriptive Statistics According to Type of Contract

\begin{tabular}{lrrrrrrr}
\hline Contract & N & Age & Male & HCE & $\begin{array}{r}\text { Share with } \\
\text { HCE }>0\end{array}$ & $\begin{array}{r}\text { HCE }^{4} \\
\text { if }>0\end{array}$ \\
\hline Low DED, FFS (baseline contract) & \multirow{2}{*}{34,053} & 55 & 0.4 & 4,610 & 0.88 & 5,230 \\
& & $(18)$ & $(0.49)$ & $(8,961)$ & $(0.33)$ & $(9,378)$ \\
Medium DED, FFS & 31,573 & 54 & 0.43 & 3,229 & 0.81 & 3,908 \\
& & $(16)$ & $(0.49)$ & $(6,962)$ & $(0.38)$ & $(7,522)$ \\
High DED, FFS & \multirow{2}{*}{38,386} & 45 & 0.54 & 1,057 & 0.57 & 1,804 \\
& & $(13)$ & $(0.5)$ & $(3,593)$ & $(0.49)$ & $(4,580)$ \\
Low DED, IPA & \multirow{2}{*}{4,942} & 54 & 0.44 & 2,933 & 0.85 & 3,427 \\
& & $(17)$ & $(0.5)$ & $(5,999)$ & $(0.35)$ & $(6,355)$ \\
Medium DED, IPA & \multirow{2}{*}{1,134} & 49 & 0.45 & 1,686 & 0.78 & 2,121 \\
& & $(15)$ & $(0.45)$ & $(3,654)$ & $(0.41)$ & $(3,999)$ \\
High DED, IPA & \multirow{2}{*}{3,598} & 43 & 0.56 & 834 & 0.58 & 1,415 \\
& & $(12)$ & $(0.56)$ & $(3,431)$ & $(0.49)$ & $(4,368)$ \\
\hline
\end{tabular}

$\mathrm{N}=163,686, \mathrm{CHF} 1 \approx \mathrm{EUR} 0.66$, Standard deviations in parentheses $\mathrm{DED}=$ Deductible, IPA $=$ Independent Practitioners Association

Turning to the supply-side cost sharing alternative (lower half of table 2), one notices that the high-deductible variant is again characterized by a comparatively low mean age and a higher share of men. Average HCE is CHF 828 or 28 percent of the low-deductible benchmark of CHF 2,934. The share of individuals with positive HCE is 58 percent rather than 85 percent, while mean HCE conditional on being positive amounts to CHF 1,408 or 41 percent of the benchmark.

However, the HCE values for the IPA plans are lower throughout than for the conventional FFS plans with the same deductible level. This may well be due to changed incentives facing physicians. Similar to the United States, participating physicians (mainly general practitioners) are paid a risk-adjusted capitation payment designed to cover all services rendered or prescribed up to a threshold of CHF 10,000 per patient and year. Beyond that limit, the insurer reimburses 90 percent of cost FFS. Capitation payments are adjusted for age, gender, deductible level, hospitalization during previous year, nursing home stay during previous year, and 21 pharmaceutical cost groups. The pharmaceutical cost groups are similar to those used in the Dutch risk adjustment scheme (Lamers and Van Vliet [2003]). While the insurer does 
not impose guidelines or utilization reviews, many networks run them internally, combined with quality monitoring by independent auditors in some cases.

In order to get a preliminary indication of the extent to which the cost differences may be caused by self-selection effects, it is instructive to compare the HCE of consumers who switch to higher deductibles and IPA plans with that of those who do not (see Table 3). The switchers from a low-deductible FFS plan in 2005 to a high-deductible one had caused HCE of CHF 826, a mere 19 percent of the non-switchers. Those changing to a mediumdeductible alternative had caused HCE amounting to CHF 1,916 in 2005, or 44 percent of the non-switchers. Switchers who moved from a FFS to an IPA plan had cost CHF 1,600, or 49 percent of the stayers. These figures point to substantial self-selection effects in both demand-side and supply-side cost sharing.

Table 3: Prior-year Mean HCE of Switchers and Non-switchers

\begin{tabular}{lrrr}
\hline Switch at the start 2006 & $\begin{array}{r}\text { FFS, low DED } \\
\text { to Medium DED }\end{array}$ & $\begin{array}{r}\text { FFS, low DED } \\
\text { to High DED }\end{array}$ & $\begin{array}{r}\text { FFS(all DED) } \\
\text { to Capitated IPA }\end{array}$ \\
\hline Non-Switchers & 4,315 & 4,315 & 3,230 \\
Switchers & 1,916 & 826 & 1,597 \\
\hline
\end{tabular}

DED $=$ Deductible, IPA $=$ Independent Practitioners Association

CHF $1 \approx$ EUR 0.66 in 2006

\section{Econometric Model}

\subsection{Developing a Proxy for Unobserved Health Status}

The dataset does not contain direct information on health status such as diagnostic codes, restrictions on activities of daily living, or self-reported health. However, panel data allows to develop an indicator of health status from prior HCE (see Van Kleef et al. [2008], Lehmann and Zweifel [2004], Wolfe and Godderies [1991]). In particular, Lehmann and Zweifel show that residuals from a random-effects Tobit regression of prior HCE on exogenous variables can serve as a proxy for unobserved health.

However, in view of considerable heteroscedasticity in the dataset, the two-part model is preferable over the Tobit. The first part is a random-effects probit model predicting the probability of observing positive HCE for individual i in year t [see Eq. (1)]. The second part 
estimates the amount of $\mathrm{HCE}$ given that it is positive. The log transformation serves to reduce the skewness of the dependent variable. Equation (2) can also be justified by noting that an individual's health is to be seen relative to her age, gender, and regional peers. The present panel is unbalanced, as many individuals had positive HCE in some but not in all years. A Wooldridge test of serial correlation in the error term (Wooldridge [2002]) rejected the null hypothesis of no autocorrelation. Therefore, the feasible generalized least squares procedure proposed for unbalanced panels by Baltagi and $\mathrm{Wu}$ [1999] is applied to gain efficiency while avoiding biased estimation of standard errors. The model applied for deriving the health status proxy thus reads (all error terms assumed normally distributed),

$$
\begin{array}{r}
\operatorname{Pr}\left(H C E_{i, t}>0\right)=\Phi\left(a+\beta X_{i, t}+\alpha_{i}+v_{i, t}\right) \\
\log \left(H C E_{i, t} \mid H C E_{i, t}>0\right)=b+\theta X_{i, t}+\gamma_{i}+\epsilon_{i, t} \\
\text { with } \quad \epsilon_{i, t}=\rho * \epsilon_{i, t-1}+\xi_{i, t}
\end{array}
$$

Eqs (1) and (2) are estimated on the first three years of the dataset, ie 2003 to 2005. Explanatory variables are age, age interacted with gender, urbanization, area of residence and a year dummy to account for inflation. Estimation results are shown in appendix A. Deviations from the expected value of $\mathrm{HCE}$ are averaged over the three years in order to reduce the influence of transitory health shocks. ${ }^{5}$

\subsection{Endogeneity of Contract Choice}

Even if the proxy can sufficiently control for unobserved differences in current health status, there are additional unmeasured variables that may cause someone opting for the minimum deductible to have a great deal of HCE, resulting in an overestimation of moral hazard effects. Examples are private information about probabilities of future illness, general attitude towards medical care, and previous experience with the health care system. Ignoring these confounders will lead to omitted variable bias in the HCE equation. Terza et al. [2008] show that the

\footnotetext{
${ }^{5}$ Note that while only individuals with the baseline contract are included here, estimated coefficients will be used to predict individual HCE for the whole sample. This has the advantage that endogeneity of contract choice does not bias estimators. However, calculating the proxy including all individuals did not change the results.
} 
residuals from an equation modeling contract choice are good estimators of these confounders. Therefore, including these residuals into the HCE equation alongside observed contract choice and the proxy for latent health serves to control for unobserved confounders (two stage residual inclusion estimation, 2SRI). The 2SRI method yields also consistent estimates if the HCE equation is nonlinear. However, it requires equations for contract choice to be specified.

For identification, at least one explanatory variable in the contract choice equation must not appear in the HCE equation. Five such variables are available.

1. Baseline Premium: A high baseline premium increases the attractiveness of higherdeductible and IPA options. At the same time, there is little reason that premiums should influence health care consumption. The income effects of premiums on health care consumption are limited in the Swiss case because some 30 percent of the population (those with low-incomes) are eligible to a premium subsidy. Moreover, preliminary estimations showed that premiums do not influence $\mathrm{HCE}$ when other factors are controlled for. ${ }^{6}$

2. Absolute premium reductions for a higher-deductible or an IPA option: While higher rebates make these contract options more attractive, they do not influence health care consumption for the reasons described in 1.

3. Number of years of CSS membership: ${ }^{7}$ Long-standing members are known not to switch contracts, making them less likely to opt for a higher-deductible or an IPA option. However, loyalty is correlated with health status because consumers who develop chronic conditions face a premium hike if they sign up with another insurer for the supplementary component (which they usually prefer to have from the same insurer to avoid ambiguity as to responsibility for payment). Nevertheless, preliminary estimations showed it to be insignificant in the HCE equation when entered in combination with the health status proxy. It therefore qualifies as identifying restriction.

4. Dummy indicating a bad credit record: This may reflect lower income, which is relevant for contract choice. At the same time, a bad credit record proved unrelated to HCE,

\footnotetext{
${ }^{6}$ Premium levels were also used as identifying instruments in Schellhorn [2001].

${ }^{7}$ This variable is truncated at 1999 because retrieving data from previous years is cumbersome. There was a change in IT architecture in 1998.
} 
once the proxy for health status was included.

5. IPA officially on offer within the individual's zipcode area: This proved to be unrelated to utilization if regional differences were controlled for by dummies. However, the availability of an IPA importantly favors the choice of the corresponding option.

Modeling the choice of deductible calls for an ordered probit model, while for the choice of the IPA a probit model is sufficient. Starting with the latter for simplicity, the generalized residuals are given by Gourieroux et al. [1987]. Let $h_{i}$ be an indicator variable equal to one if the IPA plan was chosen and zero otherwise, $z_{i}$ a vector of covariates, and $\hat{\theta}$ a vector of the estimated coefficients. Then the generalized residuals are given by

$$
\hat{u}_{i}=h_{i} * \frac{\phi\left(z_{i}^{\prime} \hat{\theta}\right)}{\Phi\left(z_{i}^{\prime} \hat{\theta}\right)}+\left[1-h_{i}\right] * \frac{-\phi\left(z_{i}^{\prime} \hat{\theta}\right)}{1-\Phi\left(z_{i}^{\prime} \hat{\theta}\right)}=\frac{\left[h_{i}-\Phi\left(z_{i}^{\prime} \hat{\theta}\right)\right] \phi\left(z_{i}^{\prime} \hat{\theta}\right)}{\left[1-\Phi\left(z_{i}^{\prime} \hat{\theta}\right)\right] \Phi\left(z_{i}^{\prime} \hat{\theta}\right)},
$$

where $\Phi$ denotes the cumulative and $\phi$, the standard normal density respectively. In the same spirit, the generalized residuals for multinomial or ordered choice models have been defined by Vella [1993]. Let there be $\mathrm{i}=1 \ldots \mathrm{N}$ individuals choosing from $\mathrm{k}=1 \ldots \mathrm{K}$ ordered alternatives, and $d_{i k}$ denote an indicator function taking the value 1 if individual $\mathrm{i}$ has chosen alternative $\mathrm{k}$ and zero otherwise. Then the generalized residual is

$$
\hat{v}_{i}=\Sigma_{k=1}^{K} d_{i k} \frac{\hat{\pi}_{i k}\left[d_{i k}-\hat{\Pi}_{i k}\right]}{\left[1-\hat{\Pi}_{i k}\right] \hat{\Pi}_{i k}}
$$

with $\hat{\Pi}_{i k}$ denoting the estimated probability that individual i chooses the $\mathrm{k}$-th alternative and $\hat{\pi}_{i k}$, the estimated value of the density at that point. These two quantities are determined as follows. Let $\hat{\gamma}$ be the vector of estimated coefficients from the ordered probit and $\hat{\alpha_{k}}$, the estimated cut points with $\alpha_{0}=-\infty$, and $\alpha_{K}=\infty$. Then,

$$
\hat{\pi}_{i k}=\phi\left(\alpha_{k-1}-z_{i}^{\prime} \hat{\gamma}\right)-\phi\left(\alpha_{k}-z_{i}^{\prime} \hat{\gamma}\right) \text { and } \hat{\Pi}_{i k}=\Phi\left(\alpha_{k-1}-z_{i}^{\prime} \hat{\gamma}\right)-\Phi\left(\alpha_{k}-z_{i}^{\prime} \hat{\gamma}\right)
$$

\subsection{Specification of the Outcome Equations}

The distribution of HCE has a cumulation point at zero. Among the alternatives available for dealing with this fact, the two-part model is preferred over e.g. the Tobit model for two reasons. First, the zeros are perceived as reflecting choices rather than missing values (see 
Jones [2000]). Second, both supply- and demand-side cost sharing are known to affect the decision to use health care in a different manner than they affect the decision how much care to use.

The first part of the two-part model is often specified by probit and estimated by maximum likelihood. However, with the inclusion of the residuals of the contract choice variables, the errors are non-normally distributed and maximum likelihood is not consistent. Instead, a glm estimation with a probit link is applied. This method is consistent as long as $E(d \mid x)=$ $\Phi(x \beta)$, with $d$ denoting an indicator that equals one if $\mathrm{HCE}>0$ and zero otherwise. Normal distribution of the error terms is not required (Cameron and Trivedi [2005]). ${ }^{8}$

The specification of the second part $(H C E \mid H C E>0)$ has been discussed by Manning [1998] and Manning and Mullahy [2001] (MM hereafter). Because of the positive skewness of the dependent variable, raw-scale estimates can be imprecise even in large datasets. The $\log$ transformation is often used to mitigate skewness, with coefficients interpreted as (semi-) elasticities of the mean response. However, Manning [1998] shows that if the error variance is heteroscedastic in a way that is correlated with the covariates, these coefficients are no longer consistent elasticity estimates. Moreover, absolute savings due to cost sharing may be of interest, calling for a retransformation of predicted values.

Blough et al. [1999], MM and others suggest estimating $\ln (E[y \mid x, y>0])$ directly by a GLM procedure with a $\log \operatorname{link}($ i.e. $\ln (E[y \mid x, y>0])=x \beta)$ and an appropriate variance function. As MM point out, the GLM estimates are consistent as long as the mean function is correctly specified, but might lead to imprecise estimates if the residuals are positively skewed even after transformation to log. Following the procedure for model selection suggested by MM, we start with a consistent GLM procedure, the gamma regression. The kurtosis of the residuals on the log scale is 3.53. This creates a tradeoff between imprecision (GLM) and possible bias (OLS applied to $\ln (\mathrm{y})$ ). In this work, GLM is used because taking heteroscedasticity into account is deemed more important than precision. Given GLM, a Park test is performed to select the variance function. The estimated $\lambda$ is 1.81 , which is closest to the gamma specification.

The residuals from the contract choice equation are estimates rather than observations.

\footnotetext{
${ }^{8}$ There is no contradiction to Terza et al. [2008], who suggested estimation by non-linear least squares. Although it goes by a different name, GLM is an iteratively reweighted nonlinear least squares estimator (Hardin and Hilbe [2007]).
} 
Not accounting for this in the outcome estimation could lead to downward biased estimates of the standard errors (Heckman [1976]). Therefore, the standard errors were obtained by bootstrapping. ${ }^{9}$

\section{Results}

\subsection{Effects of Demand-Side and Supply-Side Cost Sharing}

The results for the first part of the two-part model are shown in the first three columns of Table 4. The first column pertains to the full model. The second column excludes the residuals from the contract choice equation. The third column shows a naive specification that also excludes the proxy for health status. For the variables of interest (DED, IPA), marginal effects are calculated for a representative individual (in italics below the coefficients), ie a woman at the age of 52 , living in a suburban community in the Zurich region, having the baseline contract plus accident coverage, and a supplement covering alternative medicine. The health proxy is taken at its sample average. This individual's estimated probability of positive HCE is roughly 91 percent. For interaction terms, marginal effects are calculated according to the formulas provided by Norton et al. [2004]. ${ }^{10}$

Regardless of the specification, voluntary deductibles progressively reduce the probability of positive $\mathrm{HCE}$; however, their effect is about seven times smaller in the full and restricted than in the naive specification, pointing to considerable self-selection effects based on health status. On the other hand, they cannot be said to depend on the type of plan (DED * IPA insignificant). By way of contrast, membership in an IPA is associated with a higher probability of positive HCE (significant only in the restricted model). This may be the effect of preventive services offered. For example, one large IPA hands out vouchers for free immunizations against the flu in the fall. The variable 'drugs via pharmacy' measures the percentage of drug expenditure sold by pharmacies as opposed to physicians in the individual's

\footnotetext{
${ }^{9}$ The estimation was repeated 400 times after resampling with replacement (clustered by patient). In our specific application, the bootstrapped standard errors turned out to be similar to those obtained in the original estimation.

${ }^{10}$ To be specific, let $\beta_{a}$ and $\beta_{b}$ be the coefficients of two dummies, $\beta_{a b}$ the coefficient of their interaction and $\bar{x} \beta$ the influence of all other variables at representative values. The marginal effect of the interaction term is $\Phi\left(\beta_{a}+\beta_{b}+\beta_{a b}+\bar{x}_{\prime} \beta\right)-\Phi\left(\beta_{a}+\bar{x} \prime \beta\right)-\Phi\left(\beta_{b}+\bar{x}^{\prime} \beta\right)+\Phi\left(\bar{x}^{\prime} \beta\right)$. For a dummy without interaction, the marginal effect is $\Phi\left(\beta_{a}+\bar{x} \prime \beta\right)-\Phi(\bar{x} / \beta)$. As these marginal effects are combinations of all coefficients, their standard errors are calculated by the delta method. The calculations are run in STATA using the nlcom command.
} 
Table 4: Estimation Results from the Two-part Model

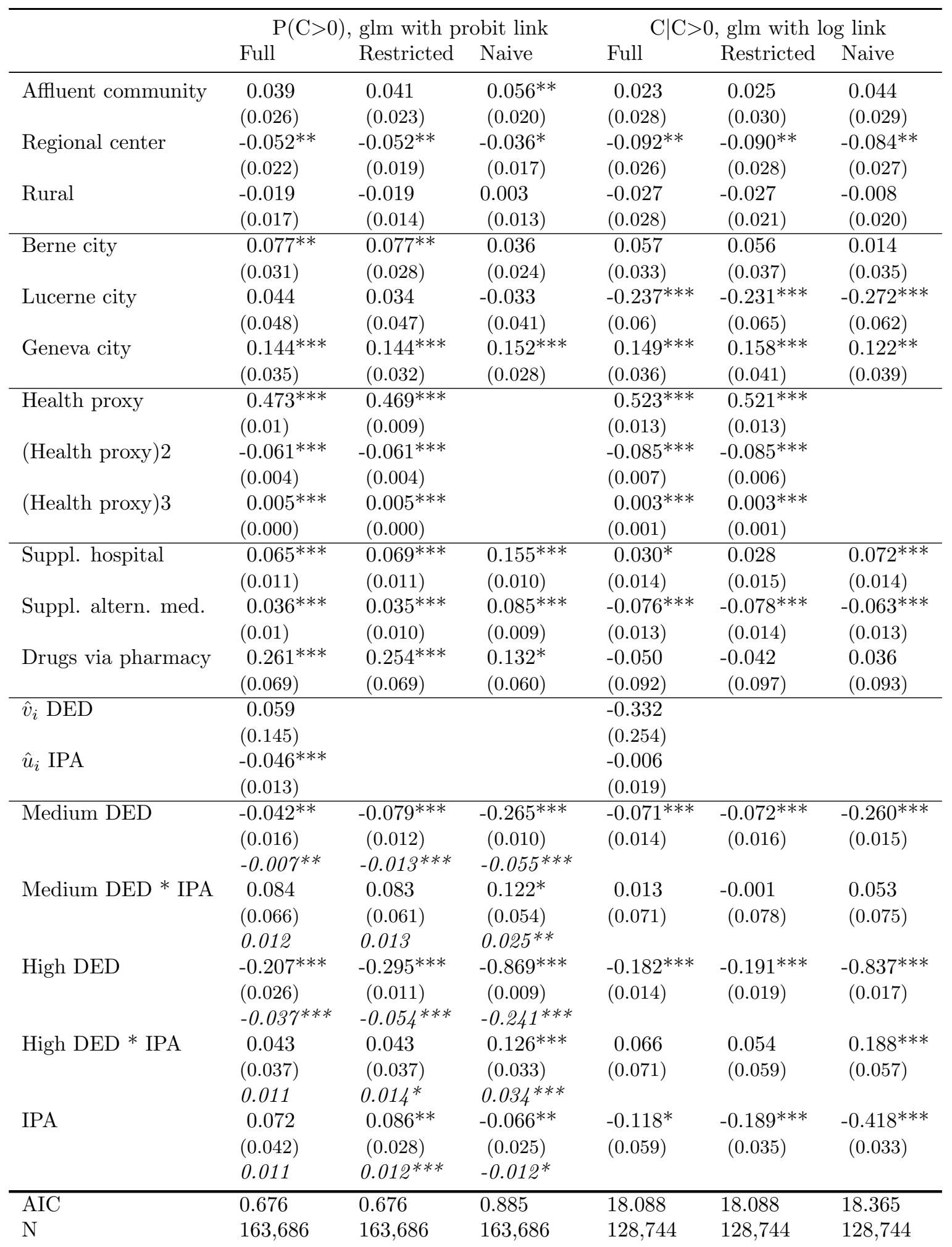

$\overline{\mathrm{DED}}=$ Deductible, IPA $=$ Independent Practitioners Association, Standard errors in parentheses, Marginal effects in italics. ${ }^{*} \mathrm{p}<0.05,{ }^{*} \mathrm{p}<0.01,{ }^{* * *} \mathrm{p}<0.001$

Additional regressors are age, gender, additional types of municipalities and areas, accident insurance. 
area of residence. Some cantons allow physicians to dispense pharmaceuticals on their own account, creating an incentive to over-prescribe. However, this fact does not seem to have an impact on HCE. ${ }^{11}$ Finally, it is noteworthy that the squared and the cubic form of the health proxy are highly significant as well. The impact of past, time-invariant health status on current expenditure does not appear to be linear over the whole distribution of HCE.

In the second part of the model, the amount of HCE is estimated (last three columns of Table 4). Higher voluntary deductibles are again found to progressively reduce HCE regardless of specification. This time, the full and restricted models point to effects that are four times smaller than according to the naive model. Type of contract does not play a role (DED * IPA insignificant). Turning to the IPA alternative, one notices a reduction of HCE amounting from 12 (full model) to 19 percent (restricted model), less than one-half of the estimate suggested by the naive specification.

\subsection{Estimating Overall Effects}

In a practical context, cost savings in actual Francs are often of interest rather than logs or probabilities. Unlike relative savings, these depend on the expenditure level of the subpopulation in question. ${ }^{12}$ The results are displayed in Table 5. For estimate (1), expected HCE according to type of contract is estimated by predicting the product of the probability of positive $\mathrm{HCE}$ and the amount of HCE. For instance, individuals with the baseline contract had expected HCE of CHF 4,320 (the reference value), while those with a high deductible combined with the IPA option had CHF 843 only. Note that the value of CHF 843 is the estimated average expenditure of the individuals who actually chose the high deductible and IPA contract. In order to estimate the expenditure of the same subpopulation assuming they had chosen the baseline contract, the dummies for deductibles and IPA plans are set to zero when predicting expected HCE. The results are shown as estimate (2) of Table 5. For example, estimate (2) for the subpopulation in the high-deductible combined with the IPA option amounts to CHF 1,111. As estimates (2) assume everybody to have chosen the baseline contract, differences from the reference value amount to pure risk-selection effects. For

\footnotetext{
${ }^{11}$ The existing literature on the issue is inconclusive. For example, Vatter and Ruefli [2003] and Trottmann [2011] find physician dispensing to lower HCE, while Beck et al. [2004] and Zweifel [1985] find a positive effect.

${ }^{12}$ If, for example, a group of very healthy individuals reduces their spending by 20 percent, the amount saved in absolute Francs is lower than if an average group does the same.
} 
individuals in the high-deductible-and-IPA option, these are estimated at CHF 3,209 (4,320 $1,111)$.

Table 5: Effects of Cost Sharing on Expected HCE

\begin{tabular}{lrrrrrr}
\hline & \multicolumn{2}{c}{ Minimum DED } & \multicolumn{2}{c}{ Medium DED } & \multicolumn{2}{c}{ High DED } \\
& FFS & IPA & FFS & \multicolumn{1}{c}{ IPA } & FFS & IPA \\
\hline Avg $\widehat{H C E}$, estimate (1) & 4,320 & 2,985 & 3,180 & 2,023 & 1,100 & 843 \\
SE & $(29)$ & $(99)$ & $(37)$ & $(159)$ & $(23)$ & $(55)$ \\
Avg $\widehat{H C E}$, estimate (2) & & 3,340 & 3,430 & 2,374 & 1,422 & 1,111 \\
SE & & $(133)$ & $(34)$ & $(124)$ & $(57)$ & $(78)$ \\
\hline Selection, (ref.) - (2) & & 980 & 891 & 1,946 & 2,898 & 3,209 \\
Moral Hazard, (2) - (1) & & 355 & 250 & 351 & 322 & 267 \\
\hline
\end{tabular}

Estimate (1): Expected HCE per contract

Estimate (2): Expected HCE of same individuals, assuming they had chosen the minimum deductible and no IPA. CHF $1 \approx$ EUR 0.66

Estimates (1) and (2) of the same column show predicted HCE for the same group of individuals, with (1) taking plan choice as observed and (2) assuming everyone to have chosen the baseline contract. Then, the difference between estimates (1) and (2) within one column is the estimated incentive effect; for high deductibles combined with IPA, it amounts to CHF $267(=1,111-843)$. Note that while this number seems rather low, it amounts to 24 percent of the predicted cost for this subpopulation assuming they were in the baseline contract. As these individuals are healthy on average, their potential for cost savings in absolute Francs is lower than that of sicker individuals. For individuals in the high deductible and FFS option, the estimated moral hazard reduction amounts to CHF $322(=1,422-1,100)$. Finally, the IPA option combined with the minimum deductible profits from moderate risk selection and achieves an absolute moral hazard reduction of CHF 355.

\subsection{Specification Tests}

In order to test the validity of our results, several alternate specifications were applied. In the first and the third column of Table 6, results are shown for a pooled glm estimation over the years 2004 - 2006 (standard errors are clustered by patient). The proxy was calculated per year using data from the respective previous year. The estimated incentive effects are stronger, likely because the incidence of chronic illness is not controlled for. Column 2 and 4 show the results of pooled estimations over the years 2005 and 2006. The proxy was calculated 
over the two previous years. With the exception of the IPA option at the first stage of the model, the estimates are close to the ones obtained by the original approach.

Finally, a fixed effects specification was used on the second part of the two-part model. ${ }^{13}$ The target variable here is the log of cost over the years 2004 - 2006. For the high deductible and the IPA, the estimated incentive effects are close to those in Table 4. For the medium deductible, the direct incentive effect is weaker and insignificant, but the interaction term with the IPA is stronger.

Table 6: Specification Tests Using Other Time Horizons or Fixed Effects

\begin{tabular}{|c|c|c|c|c|c|}
\hline \multirow{3}{*}{ Proxy Calculation } & \multicolumn{2}{|c|}{$\mathrm{P}(\mathrm{C}>0)$, glm with probit link } & \multicolumn{2}{|c|}{$\mathrm{C} \mid \mathrm{C}>0$, glm with log link } & \multirow{3}{*}{$\begin{array}{l}\text { Fixed Effects } \\
\operatorname{Ln}(\mathrm{C} \mid \mathrm{C}>0) \\
2004-06\end{array}$} \\
\hline & 1 year & 2 year & 1 year & 2 year & \\
\hline & 2004-06 & $2005-06$ & 2004-06 & $2005-06$ & \\
\hline \multirow[t]{2}{*}{ Medium DED } & $-0.137^{* * *}$ & $-0.097^{* * *}$ & $-0.117^{* * *}$ & $-0.074^{* * *}$ & -0.003 \\
\hline & $(0.007)$ & $(0.009)$ & $(0.011)$ & $(0.015)$ & $(0.014)$ \\
\hline \multirow{2}{*}{ Medium DED $*$ IPA } & 0.055 & 0.080 & -0.037 & -0.047 & $-0.154^{*}$ \\
\hline & $(0.043)$ & $(0.059)$ & $(0.060)$ & $(0.081)$ & $(0.071)$ \\
\hline \multirow[t]{2}{*}{ High DED } & $-0.441^{* * *}$ & $-0.329^{* * *}$ & $-0.265^{* * *}$ & $-0.185^{* * *}$ & $-0.149^{* * *}$ \\
\hline & $(0.008)$ & $(0.011)$ & $(0.016)$ & $(0.021)$ & $(0.017)$ \\
\hline \multirow[t]{2}{*}{ High DED * IPA } & $0.093^{* * *}$ & $0.070^{*}$ & -0.041 & -0.077 & -0.091 \\
\hline & $(0.024)$ & $(0.030)$ & $(0.048)$ & $(0.056)$ & $(0.049)$ \\
\hline \multirow[t]{2}{*}{ IPA } & $-0.100 * * *$ & $-0.061^{*}$ & $-0.284^{* * *}$ & $-0.201^{* * *}$ & $-0.108^{*}$ \\
\hline & $(0.022)$ & $(0.029)$ & $(0.048)$ & $(0.060)$ & $(0.043)$ \\
\hline $\mathrm{N}$ & 475,107 & 325,442 & 372,091 & 256,166 & 372,091 \\
\hline
\end{tabular}

Additional regressors are equal to those in Table 4 . The health proxy was calculated yearly. Standard errors in parentheses, ${ }^{*} \mathrm{p}<0.05,{ }^{*} \mathrm{p}<0.01,{ }^{* * *} \mathrm{p}<0.001$

The switchers allow for an alternate testing strategy. Here, the target variable is the difference in cost from one year to the next. This difference is symmetrically distributed, so the estimations were run by an untransformed linear random effects model. As the estimated incentive effects in Table 5 are calculated relative to the baseline contract, they are comparable to the effect of a switch away from the baseline. Column 1 of Table 7 shows the number of observations for each type of switch, column 2 shows the estimated coefficients. In the medium and high deductible case, the estimates are in the range of those in Table 5. For the IPA options, the estimated effects in the switcher regression are smaller than those in Table 5. The standard errors in the switcher estimation are larger, likely because of the limited number of

\footnotetext{
${ }^{13}$ It was not applied to the first part because of the incidental parameters problem (see Section 2).
} 
switchers observed.

Table 7: Analysis of the Switchers

\begin{tabular}{lrlr}
\hline Switch From Baseline Contract & Number of Switchers & \multicolumn{2}{l}{ Random Effects Regression } \\
& & Coefficient & SE \\
\hline .. to DED med, FFS & 2,139 & -212.99 & $(130.06)$ \\
... to DED high, FFS & 13,503 & $-387.99^{* * *}$ & $(53.27)$ \\
... to DED low, IPA & 1,905 & -260.27 & $(111.07)$ \\
... to DED med, IPA & 88 & -189.229 & $(454.35)$ \\
$\ldots$ to DED high, IPA & 805 & -219.12 & $(161.17)$ \\
\hline
\end{tabular}

Additional regressors are equal to those in Table 4.

Standard errors in parentheses, ${ }^{*} \mathrm{p}<0.05,{ }^{* *} \mathrm{p}<0.01,{ }^{* * *} \mathrm{p}<0.001$.

\subsection{Which Types of Medical Care Are Most Affected by Cost Sharing?}

In order to analyze the effects of cost sharing in more detail, the estimation technique described in section 5 is applied to general practitioners' services, specialists, drugs, physical therapy, outpatient hospital services and inpatient hospital services. Note that all services rendered by qualified specialists are attributed to 'specialist medicine' although qualified specialists might also provide primary care services. In line with the four part model advocated by Duan et al. [1982], the probability of observing positive hospital expenditure is estimated only for the individuals with positive outpatient expenditure.

A medium deductible significantly reduces the probability of GPs, drugs and hospital outpatient services. For specialized medicine, a significantly positive influence is estimated, which contradicts intuition. A high deductible reduces the probability of all types of care. The impact on hospital inpatient care is surprising as most patients have used up the deductible before entering a hospital. However, patients with high deductibles are less likely to enter the whole process of diagnostic testing and procedures, which also reduces the probability of being hospitalized. The IPA-option is found to encourage the use of GP, specialists and drugs but discourages the use of physical therapy and hospital services.

Turning to the second part of the two-part model, a medium deductible has a negative impact on the utilization of GP services and drugs. A high deductible reduces the amount of GP care, specialist medicine and drugs. Finally, the IPA option reduces the expenditure for almost all types, most strongly for specialists and drugs. As fees of specialists and hospitals 
Table 8: Incentive Effects per Type of Care

\begin{tabular}{|c|c|c|c|c|c|c|c|}
\hline & & GP & Specialist & Drugs & $\begin{array}{l}\text { Physical } \\
\text { Therapy }\end{array}$ & $\begin{array}{l}\text { Hospital } \\
\text { Outpatient }\end{array}$ & $\begin{array}{l}\text { Hospital } \\
\text { Inpatient }\end{array}$ \\
\hline \multirow[t]{4}{*}{ DED Med } & $\mathrm{P}(\mathrm{C}>0)$ & $-0.034^{* * *}$ & $0.024^{*}$ & $-0.076^{* * *}$ & 0.004 & $-0.019^{*}$ & -0.005 \\
\hline & & $(0.010)$ & (0.009) & $(0.012)$ & $(0.010)$ & $(0.009)$ & $(0.012)$ \\
\hline & $\mathrm{C} \mid \mathrm{C}>0$ & $-0.048^{* * *}$ & -0.018 & $-0.090^{* * *}$ & -0.026 & -0.045 & -0.045 \\
\hline & & $(0.008)$ & $(0.013)$ & $(0.020)$ & $(0.015)$ & $(0.026)$ & $(0.026)$ \\
\hline \multirow[t]{4}{*}{ DED High } & $\mathrm{P}(\mathrm{C}>0)$ & $-0.270 * * *$ & $-0.143^{* * *}$ & $-0.340 * * *$ & $-0.107 * * *$ & $-0.092^{* * *}$ & $-0.039^{* *}$ \\
\hline & & $(0.019)$ & $(0.019)$ & $(0.023)$ & $(0.024)$ & $(0.020)$ & $(0.015)$ \\
\hline & $\mathrm{C} \mid \mathrm{C}>0$ & $-0.154^{* * *}$ & $-0.087^{* * *}$ & $-0.137^{* * *}$ & 0.004 & 0.039 & -0.050 \\
\hline & & $(0.011)$ & $(0.018)$ & $(0.038)$ & $(0.021)$ & $(0.039)$ & $(0.036)$ \\
\hline \multirow[t]{4}{*}{ IPA } & $\mathrm{P}(\mathrm{C}>0)$ & $0.088^{* * *}$ & $0.103^{* *}$ & $0.087^{*}$ & $-0.049^{* *}$ & $-0.058^{* * *}$ & $-0.066^{* *}$ \\
\hline & & $(0.016)$ & $(0.032)$ & $(0.037)$ & $(0.018)$ & $(0.016)$ & $(0.022)$ \\
\hline & $\mathrm{C} \mid \mathrm{C}>0$ & $-0.084^{* * *}$ & $-0.153^{* * *}$ & $-0.156^{* *}$ & $-0.058^{*}$ & -0.088 & $-0.116^{*}$ \\
\hline & & $(0.016)$ & $(0.023)$ & $(0.053)$ & $(0.029)$ & $(0.046)$ & $(0.046)$ \\
\hline \multirow[t]{2}{*}{$\mathrm{AIC}$} & $\mathrm{P}(\mathrm{C}>0)$ & 1.042 & 1.109 & 0.822 & 0.820 & 1.061 & 0.614 \\
\hline & $\mathrm{C} \mid \mathrm{C}>0$ & 14.41 & 15.31 & 15.233 & 15.254 & 16.043 & 19.751 \\
\hline \multirow[t]{2}{*}{$\mathrm{N}$} & $\mathrm{P}(\mathrm{C}>0)$ & 163,686 & 163,686 & 163,686 & 163,686 & 163,686 & 128,744 \\
\hline & $\mathrm{C} \mid \mathrm{C}>0$ & 101,265 & 86,208 & 112,941 & 28,241 & 46,923 & 17,205 \\
\hline
\end{tabular}

Additional regressors are equal to those in Table 4

Standard errors in parentheses, ${ }^{*} \mathrm{p}<0.05,{ }^{* *} \mathrm{p}<0.01,{ }^{* * *} \mathrm{p}<0.001$

are regulated to be equal in the FFS and the IPA sector, the expenditure effect is due to a reduction in quantity only. ${ }^{14}$

\section{Discussion}

The aim of this section is to discuss the policy implications of our results, relating them to recent literature. A first surprising result is that the estimated absolute cost reduction due to a deductible of CHF 500 rather than 300 (CHF 250, see Table 5 again) exceeds the maximum increase in out-of-pocket expenditure $($ CHF $200=500-300)$. This is confirmed by two other recent studies using Swiss data from earlier years when the minimum deductible was CHF 230 and the next lowest CHF 400 (see Table 9).

\footnotetext{
${ }^{14}$ In order to validate our results, we reestimated the second part of the model by ols on log expenditure. The estimates are close and equal in sign. The only exception it the coefficient for hospital outpatient services, which is significantly negative in the OLS estimation. It is not a priori clear which is more plausible for this heterogenous patient group. Some patients are chronically ill and in need of repeated procedures (suggesting no effect), while others visit the emergency room for relatively minor ailments (where deductibles might well be effective).
} 
Table 9: Estimated Deductible Effects in Earlier Papers

\begin{tabular}{lrrrrr}
\hline \multicolumn{7}{c}{ Van Kleef et al. [2008] } \\
Deductible levels (FFS, 2003) & 230 & 400 & 600 & 1,200 & 1,500 \\
Estimated Moral Hazard Reduction & 3 & 382 & 443 & 276 & 318 \\
\hline Gardiol et al. [2005] & 230 & 400 & 600 & 1,200 & 1,500 \\
Deductible levels (FFS, 2000) & 697 & 512 & 306 & 62 & 0 \\
\hline Estimated Moral Hazard Reduction & 690
\end{tabular}

CHF $1 \approx$ EUR 0.66

Van Kleef et al. [2008] estimate that raising the deductible from CHF 230 to 400 would serve to reduce expenditure by CHF 382, exceeding the maximum increase in out-of-pocket expenditure by CHF 212. Gardiol et al. [2005] take the maximum deductible of CHF 1,500 as the point of reference and calculate the incentive effects from there. The transition from the medium deductible of CHF 400 to the minimum of CHF 230 is estimated to generate 'true' savings of CHF 185 (=697-512), which again exceeds the out-of-pocket difference of CHF 170.

The second point relates to risk adjustment (RA). Note from Table 6 that estimated moral hazard reductions not only fall far short of gross differences in expected HCE as indicated by estimates (1) but are markedly plan specific. Van Kleef et al. [2008] and Van Kleef et al. [2006] discuss the challenges this varying mix of risk-selection and moral hazard effects poses to regulators in a system which community rating and RA. The issue is the extent to which insurers should be allowed to pass on gross savings to consumers. The appropriate amount seems to be the amount of 'true' savings net of risk-selection effects. Yet, Van Kleef et al. [2006] show that if only very low risks opt for higher deductibles at first, premium reductions reflecting 'true' savings are too small to create incentives for these options. ${ }^{15}$ As a remedy, it is beneficial not to entirely net out risk-selection effects for determining allowable premium reductions. Empirical evidence by Van Kleef et al. [2008] shows that the current RA schemes of the Netherlands and Switzerland do leave room for risk-selection effects in the premium reductions.

\section{Conclusions}

Managed competition in social health insurance aims at creating incentives for insurers to increase efficiency and respond to consumer preferences while preserving solidarity between

\footnotetext{
${ }^{15}$ This reflects the Swiss experience after the introduction of voluntary deductibles in 1996.
} 
high- and low-risk types (Van de Ven et al. [2007]). Therefore, it is important to know whether contractual innovations such as deductibles or capitated IPA plans achieve true cost savings rather than merely serving as a means for risk selection. This research measures and compares the impacts of demand-side cost sharing (through voluntary deductibles) and supply-side cost sharing (through prepaid IPA plans) on total health care expenditure (HCE), controlling for risk-selection effects. The data comes from a large panel of Swiss adults covering the years 2003 to 2006. Since unobserved health status influences both contract choice and HCE, a proxy is constructed from HCE during the first three years of the observation period, complemented by the residuals from the contract choice equation [two stage residual inclusion method (Terza et al. [2008])].

Higher annual deductibles and IPA plans are both found to achieve marked reductions of moral hazard. An increase in the deductible by CHF 200 (some EUR 133) decreases the estimated probability of positive HCE during a given year by almost 1 percentage point, while the IPA plan is associated with an increase of about 1.1 points. The amount of positive HCE is reduced by some 7 percent and 12 percent, respectively. Increasing the deductible by CHF 700 (some EUR 466) reduces the probability of reporting HCE by about 3.7 percent and the amount of positive HCE by about 18 percent.

Still, this research is subject to several limitations. First, since the data set only comprises individuals who were with one and the same insurer from 2003 - 2006, it fails to measure riskselection effects associated with changes between competing insurers. Second, even 'within' risk-selection effects may not be controlled for perfectly. There is no guarantee that the HCE equation is correctly specified for the three preceding years, yielding residuals that serve as good proxies for unobserved health. The same caveat applies to the residuals of the contract choice equations. Thus, estimates of expected HCE reductions achieved by higher deductibles and IPA plans could still be biased. Third, the effects of the deductible option contain price and income effects. As shown by Nyman [1999] only the former should be counted as inefficient consumption. Finally, results relating to IPA plans have limited generality as long as they cannot be related in detail to the incentives faced by participating health care providers.

Nevertheless, the findings permit to draw the conclusion that allowing insurers to offer plans with both demand-side and supply-side cost sharing reduced spending in Swiss social health insurance. After controlling for risk-selection effects, both variants are estimated to 
achieve marked reductions in moral hazard that can be passed on to consumers in the guise of premium reductions without jeopardizing insurers' solvency. 


\section{Appendices}

\section{A Estimating a Proxy for Health Status}

Table 10: Results from the Random Effects Estimation

\begin{tabular}{|c|c|c|}
\hline & $\begin{array}{l}\mathrm{P}\left(C_{i t}>0\right) \\
\text { Random Effects Probit }\end{array}$ & $\begin{array}{l}C_{i t} \mid C_{i t}>0 \\
\text { Random Effects AR (1) }\end{array}$ \\
\hline Greater Metropolitan Area & $\begin{array}{l}0.018 \\
(0.021)\end{array}$ & $\begin{array}{l}-0.077^{* * *} \\
(0.010)\end{array}$ \\
\hline Affluent Community & $\begin{array}{l}0.101^{*} \\
(0.041)\end{array}$ & $\begin{array}{l}-0.069^{* * *} \\
(0.020)\end{array}$ \\
\hline Regional Center & $\begin{array}{l}-0.083^{* *} \\
(0.032)\end{array}$ & $\begin{array}{l}-0.203^{* * *} \\
(0.017)\end{array}$ \\
\hline Rural, mainly industrial & $\begin{array}{l}-0.070^{* *} \\
(0.025)\end{array}$ & $\begin{array}{l}-0.173^{* * *} \\
(0.013)\end{array}$ \\
\hline Rural, agriculture & $\begin{array}{l}-0.171^{* * *} \\
(0.028)\end{array}$ & $\begin{array}{l}-0.209^{* * *} \\
(0.015)\end{array}$ \\
\hline Berne city & $\begin{array}{l}0.088^{* *} \\
(0.033)\end{array}$ & $\begin{array}{l}0.037^{*} \\
(0.016)\end{array}$ \\
\hline Lucerne city & $\begin{array}{l}-0.059^{*} \\
(0.028)\end{array}$ & $\begin{array}{l}-0.241^{* * *} \\
(0.014)\end{array}$ \\
\hline Geneva city & $\begin{array}{l}0.396^{* * *} \\
(0.042)\end{array}$ & $\begin{array}{l}0.385^{* * *} \\
(0.019)\end{array}$ \\
\hline 2004 & $\begin{array}{l}0.007 \\
(0.012)\end{array}$ & $\begin{array}{l}0.091^{* * *} \\
(0.005)\end{array}$ \\
\hline 2005 & $\begin{array}{l}0.017 \\
(0.012)\end{array}$ & $\begin{array}{l}0.089^{* * *} \\
(0.005)\end{array}$ \\
\hline Constant & $\begin{array}{l}1.928^{* * *} \\
(0.036)\end{array}$ & $\begin{array}{l}6.911^{* * *} \\
(0.018)\end{array}$ \\
\hline $\mathrm{N}$ & 253,653 & 218,208 \\
\hline $\begin{array}{l}\alpha \\
\rho\end{array}$ & .691 & $\begin{array}{l}.531 \\
.090\end{array}$ \\
\hline
\end{tabular}

Additional regressors are age, gender and more areas.

Standard errors in parentheses, ${ }^{*} \mathrm{p}<0.05,{ }^{* *} \mathrm{p}<0.01,{ }^{* * *} \mathrm{p}<0.001$

$\rho$ Estimated autocorrelation coefficient

$\alpha$ Fraction of error variance due to individual-specific term 


\section{B Predicting Contract Choice}

Table 11: Estimation of Contract Choice in 2006

\begin{tabular}{|c|c|c|}
\hline & $\begin{array}{l}\text { Choice of Deductible } \\
\text { Ordered Probit }\end{array}$ & $\begin{array}{l}\text { Choice of prepaid IPA } \\
\text { Probit }\end{array}$ \\
\hline \multirow[t]{2}{*}{ Health proxy } & $-0.341^{* * *}$ & $-0.219^{* * *}$ \\
\hline & $(0.006)$ & $(0.012)$ \\
\hline \multirow[t]{2}{*}{$(\text { Health proxy })^{2}$} & $0.042^{* * *}$ & $0.055^{* * *}$ \\
\hline & $(0.003)$ & $(0.005)$ \\
\hline \multirow[t]{2}{*}{$(\text { Health proxy })^{3}$} & $-0.002^{* * *}$ & $-0.004^{* * *}$ \\
\hline & $(0.000)$ & $(0.001)$ \\
\hline \multirow[t]{2}{*}{ Bad credit record } & $-0.274^{* * *}$ & $-0.359^{* * *}$ \\
\hline & $(0.014)$ & $(0.027)$ \\
\hline \multirow[t]{2}{*}{ Years of CSS membership since 1999} & $-0.042^{* * *}$ & $-0.052^{* * *}$ \\
\hline & $(0.004)$ & $0.007)$ \\
\hline \multirow[t]{2}{*}{ Baseline premium } & $0.018^{* * *}$ & $0.005 * * *$ \\
\hline & $(0.002)$ & $(0.001)$ \\
\hline \multirow[t]{2}{*}{ Premium reduction for medium DED } & 0.008 & \\
\hline & $(0.010)$ & \\
\hline \multirow[t]{2}{*}{ Premium reduction for high DED } & $0.015^{* * *}$ & \\
\hline & $(0.003)$ & \\
\hline \multirow[t]{2}{*}{ Premium reduction for IPA } & & $0.013^{* * *}$ \\
\hline & & $(0.001)$ \\
\hline \multirow[t]{2}{*}{ IPA operational in zipcode area } & & $1.436^{* * *}$ \\
\hline & & $(0.058)$ \\
\hline \multirow[t]{2}{*}{ Constant } & & $-3.926^{* * *}$ \\
\hline & & $(0.287)$ \\
\hline Cut points & $7.145 / 7.823$ & \\
\hline Log likelihood & $-140,618$ & $-27,297$ \\
\hline Number of observations & 163,686 & 163,686 \\
\hline
\end{tabular}

Additional regressors are age, gender, types of municipalities and areas, accident insurance, supplemental insurance.

Standard errors in parentheses, ${ }^{*} \mathrm{p}<0.05,{ }^{*} \mathrm{p}<0.01,{ }^{* * *} \mathrm{p}<0.001$ 


\section{References}

K. Arrow. Uncertainty and the welfare economics of medical care. American Economic Review, 53:941-973, 1963.

B. Baltagi and P. Wu. Unequally spaced panel data regressions with ar(1) disturbances. Econometric Theory, 15:814 - 823, 1999.

K. Beck, U. Kunze, and W. Oggier. Selbstdispensation: Kosten treibender oder Kosten daempfender Faktor? (Drug dispensing by physicians: Cost increasing or cost decreasing?). Managed Care, 6:5-8, 2004.

D. Blough, C. Madden, and M. Hornbrook. Modeling risk using generalized linear models. Journal of Health Economics, 18:153 - 171,, 1999.

A. Cameron and P. Trivedi. Microeconometrics. Cambridge University Press, New York, NY 10013-2473, USA, 1 edition, 2005.

A. Cameron, P. Trivedi, F. Milne, and J. Piggott. A microeconometric model of the demand for health care and health insurance in australia. Review of Economic Studies, 55:85 - 106,, 1988.

J. Cardon and I. Hendel. Asymmetric information in health insurance: Evidence from the national medical expenditure survey. RAND Journal of Economics, 32:408 - 427,, 2001.

G. Chamberlain. Analysis of covariance with qualitative data. Review of Economic Studies, $47: 225-236,, 1980$.

P. Chiappori, F. Durand, and P. Geoffard. Moral hazard and the demand fpr physician services. European Economic Review, 42:499 - 511,, 1998.

P. Deb and P. Trivedi. Provider networks and primary-care signups: Do they restrict the use of medical services? Health Economics, 18:1361 - 1380, 2009.

P. Deb, M. Munkin, and P. Trivedi. Bayesian analysis of the two-part model with endogeneity: Application th health care expenditure. Journal of Applied Econometrics, 21:1081-1099, 2006. 
R. Dowd, R. Feldman, S. Cassou, and M. Finch. Health plan choice and utilization of health care services. The Review of Economics and Statistics, 73:85 - 93, 1991.

N. Duan, W. Manning, C. Morris, and J. Newhouse. A comparision of alternative models for the demand for medical care. The Rand Publication Series, R-2754-HHS, 1982.

M. Eichner. The demand for medical care: What people pay does matter. AER Papers and Proceedings, 88:117-121, 1998.

R. Ellis and T. McGuire. Supply-side and demand-side cost sharing in health care. Journal of Economic Perspectives, 7:135-151, 1993.

A. Enthoven. Consumer-choice health plan. The New England Journal of Medicine, 12;13: 650-658 and 709-720, 1978.

L. Gardiol, P. Geoffard, and C. Grandchamp. Separating selection and incentive effects in health insurance. PSE Working Papers 2005-38, 2005.

L. Gardiol, P. Geoffard, and C. Grandchamp. Selection and incentive effects: An econometric study of swiss health-insurance claims data. In P. Chiappori and C. Gollier, editors, Competitive Failures in Insurance Markets, pages 81 - 95. MIT Press, Cambridge, MA, 2006.

M. Gerfin and M. Schellhorn. Nonparametric bounds on the effect of deductibles in health care insurance on doctor visits - swiss evidence. Health Economics, 15:1011-1020, 2006.

C. Gourieroux, A. Monfort, E. Renault, and A. Trogon. Generalized residuals. Journal of Econometrics, 34:5-32, 1987.

J. Hardin and J. Hilbe. Generalized Linear Models and Extensions. College Station: Stata Press, 2nd edition, 2007.

J. Heckman. The common structure of statistical models of truncation, sample selection and limited dependent variables and a simple estimator for such models. In NBER, editor, Annals of Economic and Social Measurement, pages 120 - 137. NBER, New York, 1976.

A. Jones. Health econometrics. In P. Newhouse and A. Culyer, editors, Handbook of Health Economics, pages 265-344. Elsevier, Amsterdam, 2000. 
L. Lamers and R. Van Vliet. Health-based risk adjustment: Improving the pharmacy-based cost group model to reduce gaming possibilies. The European Journal of Health Economics, 4:107-114, 2003.

T. Lancaster. The incidental parameter problem since 1948. Journal of Econometrics, 95:391 $-413,2000$.

L. Lee. Unionism and wage rates: A simultaneous model with qualitative and limited dependent variables. International Economic Review, 23:415-433, 1978.

H. Lehmann and P. Zweifel. Innovation and risk selection in deregulated social health insurance. Journal of Health Economics, 23:997-1012, 2004.

W. Manning. The logged dependent variable, heteroscedasticity, and the retransformation problem. Journal of Health Economics, 17:283 - 295, 1998.

W. Manning and J. Mullahy. Estimating log models: To transform or not to transform? Journal of Health Economics, 20:461 - 494, 2001.

W. Manning, J. Newhouse, N. Duan, E. Keeler, and A. Leibowitz. Health insurance and the demand for medical care: Evidence from a randomized experiment. The American Economic Review, 77:251 - 277, 1987.

T. McGuire. Physician agency. In C. AJ and N. JP, editors, Handbook of Health Economics, pages 410-459. Elesevier, Amsterdam, 2000.

J. Newhouse. Pricing the Priceless: A Health Care Conundrum. MIT Press, Cambridge, 2002.

E. Norton, H. Wang, and C. Ai. Computing interaction effects and standard errors in logit and probit models. The Stata Journal, 4:154 - 167, 2004.

J. Nyman. The economics of moral hazard revisited. Journal of Health Economics, 18:811 824, 1999.

OECD. Switzerland. Technical report, OECD Reviews of Health Systems, 2006. 
M. Schellhorn. The effect of variable health insurance deductibles on the demand for physician visits. Health Economics, 10:441 - 456, 2001.

J. Terza, A. Basu, and P. Rathouz. Two-stage residual inclusion estimation: Addressing endogeneity in health econometric modeling. Journal of Health Economics, 27:531-543., 2008.

M. Trottmann. Prescribers' financial incentives and health care expenditure. Working Paper, Department of Economics, University of Zuerich, 2011.

W. Van de Ven, K. Beck, C. Van de Voorde, J. Wasem, and I. Zmora. Risk adjustment and risk selection in europe: Six years later. Health Policy, 83:162 - 179, 2007.

R. Van Kleef, W. Van de Ven, and R. Van Vliet. Premium rebate in exchange for a voluntary deductible in social health insurance with risk equalization: Community-rated or risk rated? Journal of Risk and Insurance, 73:529 - 550, 2006.

R. Van Kleef, K. Beck, W. Van de Ven, and R. Van Vliet. Risk equalization and voluntary deductibles: A complex interaction. Journal of Health Economics, 27:427 - 443, 2008.

A. Vatter and C. Ruefli. Do political factors matter for health care expenditure? A comparative study of swiss cantons. Journal of Public Policy, 23:301-323, 2003.

F. Vella. A simple estimator for simultaneous models with censored endogenous regressors. International Economic Review, 34:441 - 457, 1993.

F. Vella and M. Verbeek. Whose wages do unions rise?a dynamic model of unionism and wage rate determination for young men. Journal of Applied Econometrics, 13:163 - 183, 1998.

F. Vella and M. Verbeek. Two-step estimation of panel data models with censored endogenous variables and selection bias. Journal of Econometrics, 90:239 - 263, 1999.

A. Werblow, S. Felder, and P. Zweifel. Population ageing and health care expenditures: A school of 'red herrings'? Health Economics, 16:1109 - 1126, 2007.

R. Winkelmann. Co-payments for prescription drugs and the demand for doctor visits evidence from a natural experiment. Health Economics, 13:1081 - 1089, 2004. 
J. Wolfe and J. Godderies. Adverse selection, moral hazard, and wealth effects in the medigap insurance market. Journal of Health Economics, 10:433 - 459, 1991.

J. Wooldridge. Econometric Analysis of Cross Section and Panel Data. MIT Press, Cambridge MA, 2002.

R. Zeckhauser. Medical insurance: A case study of the tradeoff between risk spreading and appropriate incentives. Journal of Economic Theory, 2:10 - 26, 1970.

P. Zweifel. Technology in ambulatory medical care: Cost increasing or cost saving? Social Science and Medicine, 21:1139 - 1151, 1985.

P. Zweifel and W. Manning. Moral hazard. In C. AJ and N. JP, editors, Handbook of Health Economics, pages 410-459. Elesevier, Amsterdam, 2000. 